\title{
STABILITY ANALYSIS OF MATHEMATICAL MODEL OF A RELAPSE TUBERCULOSIS INCORPORATING VACCINATION
}

\author{
Odetunde, $\mathrm{O}^{1}$, Ibrahim, M. $\mathrm{O}^{2}$
}

1,2. Department of Mathematics, University of Ilorin, Kwara State, Nigeria.

*Corresponding author: odetunde.o@unilorin.edu.ng

\section{Article Info}

Received: 20 December 2020

Accepted: 15 March 2021
Revised: 03 March 2021

Available online: 15 October 2021

\begin{abstract}
A general SIQRM epidemic model with vaccination and relapse possibility is proposed for analysis in this work. The idea behind the proposed model is to check the effect of immunity obtained from vaccine or treatment, quarantine effect as well as waning effect of immunity on the transmission rate of Tuberculosis within a population that is subjected to proper education without restricted access. Some other infectious diseases in this category include measles and Ebola. Two equilibrium states of the proposed model are obtained as well as the effective reproduction number $\left(R_{e f f}\right)$. Stability analysis of the model at the Infection Free Equilibrium (I.F.E) state is established on the condition that $R_{\text {eff }}<1$. Numerical simulation for the general SIQRM model was done using specific data for Tuberculosis disease and the result shows that proper education, vaccination and early diagnosis of an infectious individual for quarantining is an efficient way by which the spread of Tuberculosis can be reduced in the population while adequate medical attention yield better result for detected cases.
\end{abstract}

Keywords: Epidemic Model, Relapse, Immunity, Quarantine Effect, Simulation.

MSC2010:92B05

\section{Introduction}

General interest in infectious diseases and their control are fueled by discovery of new infectious disease, frequent re-appearance of plague and emergence of both preventive and therapeutic antibiotics to curb, manage and possibly eradicate the threat of such an infection [2]. Many fundamental concepts and principles to model disease transmission exist and have been built upon by several researchers to address specific infection dynamics. The most elementary of such models are the $S-I-R ; S-I-S$; and $S-E-I-R$ with $S$ as Susceptible, $I$ as Infectious, $R$ as Recovered and $\mathrm{E}$ as Exposed. In this work, a generalized $S-I-Q-R-M$ model is presented for analysis where $S, I, R$ retain their meaning and $Q$ denotes Quarantine and $M$ denotes Immunity. Though, the generalized $S-I-Q-R-M$ model is scarcely used in literature, it however can be of immense importance in predicting the course of an infection as well as proffering effective and efficient ways by which an infection in this category can be treated and eliminated. Some of the infection 
in this category includes Tuberculosis, Lassa Fever, Measles and Ebola. These infections requires high level of vigilance when they enter a population of susceptible individuals. The earlier these diseases are diagonized from an individual, the better for the entire population if such an individual is quarantined. In this work, tuberculosis shall be used as a case study to show the effect of early detection, quarantine, vaccination and treatment of an infected individual on the overall epidemic of the disease within the host population. These diseases have high relapse rate if the infectious individual do not complete his dosage for treatment appropriately. Some basic definition of terms used in this work are discussed below.

Quarantine involves separation of people with restriction of access of susceptible individuals who were exposed to a contagious disease in order to check for infectiousness [9]. It is a measure that is always taken to prevent the spread of a highly infectious disease like tuberculosis and Ebola from spreading within a population. Quarantining an exposed/infected individual cannot be taken to mean stigmatization, it is just a necessary precaution taken to reduce the spread of such disease in the community.

Similarly, immunity to a disease is defined as the quality or state of the body system to resist a particular disease through the presence of multi-cellular organisms known as antibodies. Sometimes, immunity can be obtained through vaccination, treatment and personal hygiene. Vaccination offers temporary immunity which can sometimes last for a lifetime if contact with infection is minimized to susceptible population. However, obtaining a hundred percent vaccination of entire susceptible group is practically impossible due to several factors. Also, successful treatment and recovery from certain illness, like measles, offer some degree of immunity against its re-infection, thus, some therapeutic treatment to certain diseases offers immunity either temporal or permanent against such a disease.

Relapse is defined as the worsening of a clinical condition that had previously improved. This occur most times when an individual discontinued his treatment at the earliest possible time of recovery without completing his dosage [1]. Aside from non-adherence to drug prescription, another way by which relapse can occur is due to ineffectiveness of the drug being taken to cure the disease and some other biological factor. Relapse is different from re-infection because the latter has to come in contact with those infected before developing the symptoms of the infection while the former occur without any contact with infectious individual. For instance, standard treatment for active TB requires drug treatment with at least four dosages over six months period [6].

The case of re-infection of a treated illness without a second contact with the infective class is known as relapse. The dynamics of infection and re-infection differs from one illness to another. Thus, to obtain a general model, it is important to take cognizance of peculiarity of each diseases that may fall into the general category under consideration.

Tuberculosis is a contagious and infectious disease that is caused by mycobacterium tuberculosis. This disease has always been a major challenge to mankind since it was first discovered in the $1700 \mathrm{~s}$ as the white plague. It has claimed lots of human lives and it is recorded as the top killer with close to 4000 mortality cases everyday [10]. Though, it is a preventable and curable disease, millions of people continue to fall ill with $\mathrm{TB}$ each year. It was reported that an estimated 10 million individuals got infected with TB in 2019, with 7.1 million having access to TB care while about five hundred thousand developed a drug-resistance TB illness [10]. Fors et al., [6] developed and solved some TB models with some clinical characteristics that include immune system response and patient drug therapy adherence. In their work, they presented mathematical model for pharmacokinetics by using a single compartmental system with first order absorption and elimination of drugs in the plasma. Their study presented an integrated systems pharmacology model and simulation tool to support prediction of outcomes of different therapies for pulmonary tuberculosis infection. Mettle, Affi and Twumasi [11] modelled the dynamics of TB among high-burden district by using an SEIR epidemic model with demograpphy. They also considered the effect of introducing treatment at the exposed stage on the overall transmission dynamics of the infection in the region considered. They 
established that their model perform better in eight out of ten municipals considered in the region. Thus, in this work, a new generalized SIQRM model shall be used to consider the overall effect of quarantining an early detected TB carriers. Also, infant vaccination effect, effective treatment and waning of immunity from vaccination shall be considered on the overall effective reproduction number of TB under the SIQRM epidemic model.

\section{MODEL FORMULATION}

A non-linear deterministic model for the transmission dynamics of infectious diseases in the presence of immunity loss and relapse is built by dividing the total human population at time $t$, denoted by $\mathrm{N}(\mathrm{t})$ into five disjoint epidemiological sub-populations, which are, Susceptible (S), Infected (I), Quarantine (Q), Recovered (R) and Partial Immunity (M). Thus, $N(t)=S(t)+I(t)+Q(t)+R(t)+$ $M(t)$ Recruitment into susceptible compartment is assumed either through birth or immigration at the rate of $\pi$ and immunity loss rate $\alpha$, natural mortality occurs across all compartments at the rate $\mu$, susceptible are vaccinated at the rate $\chi$ while they become infected with the force of infection which is given as $\lambda=\beta_{1} I+\beta_{2} Q$ where $\beta_{1}, \beta_{2}$ are the contact with infective and quarantined classes that are sufficient to bring about new infections. Infected class is treated and recovered at the rate $\epsilon$, and the rate of progression to the quarantined class is $\gamma$. The induced death rate of infected class is $\delta_{1}$ and re-infection rate is $\eta$. The quarantined class is reduced by natural mortality rate $\mu$, induced death rate $\delta_{2}$ and the recovery rate is $\omega_{2}$. Partial immunity compartment is increased at the rate of $\omega_{1}$ as a result of immunity obtained from recovery. The flow diagram of the model is given in figure 1 below.

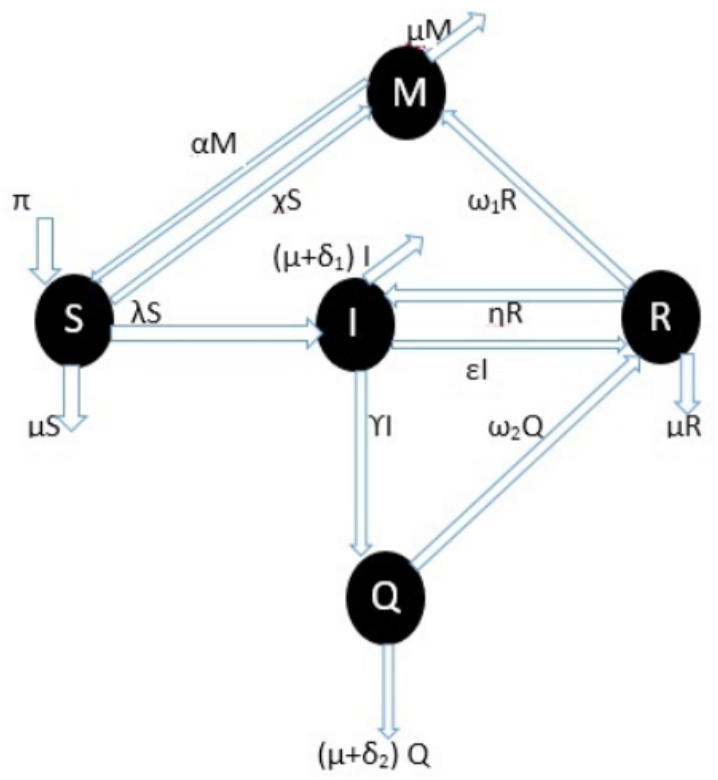

Figure 1: Pictorial representation of the Model 
The above deterministic model is govern by the following non-linear system of ordinary differential equations:

$$
\begin{aligned}
& \frac{d S}{d t}=\pi+\alpha M-\lambda S-(\mu+\chi) S \\
& \frac{d I}{d t}=\lambda S+\eta R-\left(\mu+\delta_{1}+\gamma+\epsilon\right) I \\
& \frac{d Q}{d t}=\gamma I-\left(\mu+\delta_{2}+\omega_{2}\right) Q \\
& \frac{d R}{d t}=\epsilon I+\omega_{2} Q-\left(\mu+\eta+\omega_{1}\right) R \\
& \frac{d M}{d t}=\omega_{1} R+\chi S-(\mu+\alpha) M
\end{aligned}
$$

Where the force of infection $\lambda$ is given as:

$$
\lambda=\beta_{1} I+\beta_{2} Q
$$

\section{Basic Assumptions of the Model}

The following assumptions are considered in constructing the model:

1. Recruitment is done only into the susceptible class.

2. The studied population is homogenous and varies with time.

3. Birth rate is greater than mortality rate.

4. The force of infection is expressed as $\lambda=\beta_{1} I+\beta_{2} Q$

5. Induced death rate at infected and quarantine classes are not equal.

Description of state variables, parameters value and their sources are given in table 1:

Table 1: Table of parameter value for the Model

\begin{tabular}{|c|l|c|l|}
\hline Symbol & Description & Value & Source \\
\hline$\pi$ & Recruitment rate & $\mu \times 10^{5}$ & {$[8]$} \\
\hline$\chi$ & Vaccination rate & 0.3 & Assumed \\
\hline$\alpha$ & Immunity loss & 0.5 & Assumed \\
\hline$\mu$ & mortality rate & 0.02041 & {$[8]$} \\
\hline$\gamma$ & quarantined rate & 0.4 & Assumed \\
\hline$\delta_{1}$ & Induced death rate of I-class & 0.365 & {$[8]$} \\
\hline$\delta_{2}$ & Induced death rate of Q-class & 0.365 & {$[8]$} \\
\hline$\eta$ & Relapse or re-infection rate & 0.1 & {$[8]$} \\
\hline$\omega_{2}$ & Recovery rate of quarantined class & 1.5 & {$[8]$} \\
\hline$\omega_{1}$ & Recovery rate & 0.6 & Assumed \\
\hline$\epsilon$ & Recovery rate & 0.75 & {$[8]$} \\
\hline$S(t)$ & Susceptible per time & Varied & Assumed \\
\hline$I(t)$ & Infected per time & Varied & Assumed \\
\hline$Q(t)$ & Quarantine per time & Varied & Assumed \\
\hline$R(t)$ & Recovered per time & Varied & Assumed \\
\hline$M(t)$ & Immune class per time & Varied & Assumed \\
\hline
\end{tabular}




\section{Model Solution}

Setting $c_{1}=\mu+\chi, c_{2}=\mu+\delta_{1}+\gamma+\epsilon, c_{3}=\mu+\delta_{2}+\omega_{2}, c_{4}=\eta+\mu+\omega_{1}, c_{5}=\alpha+\mu$ for compactness of computation to obtain:

$$
\begin{aligned}
& \frac{d S}{d t}=\pi+\alpha M-\lambda S-c_{1} S \\
& \frac{d I}{d t}=\lambda S+\eta R-c_{2} I \\
& \frac{d Q}{d t}=\gamma I-c_{3} Q \\
& \frac{d R}{d t}=\epsilon I+\omega_{2} Q-c_{4} R \\
& \frac{d M}{d t}=\omega_{1} R+\chi S-c_{5} M
\end{aligned}
$$

\section{Theorem 1:}

The set $D=\left\{(S, I, Q, R, M) \in \mathbb{R}_{+}^{5}: N=S+I+Q+R+M \leq \frac{\pi}{\mu}\right\}$ is closed and positively invariant and attracting with respect to the system (1)

\section{Proof:}

From (1), setting

Such that

$$
\frac{d N}{d t}=\frac{d S}{d t}+\frac{d I}{d t}+\frac{d Q}{d t}+\frac{d R}{d t}+\frac{d M}{d t}
$$

$$
\begin{gathered}
\frac{d N}{d t}=\pi-\mu N-\delta_{1} I-\delta_{2} Q \\
\frac{d N}{d t} \leq \pi-\mu N
\end{gathered}
$$

Solving the above with initial condition $N(0)=N_{0}$ to obtain

$$
N(t) \leq \frac{\pi}{\mu}+\left(N_{0}-\frac{\pi}{\mu}\right) e^{-\mu t}
$$

It is obvious that $N(t)$ approaches $\frac{\pi}{\mu}$ as $t \rightarrow \infty$, thus, it follows that the system (1) is positively invariant and attracting in domain $D$. Therefore the model is mathematically and epidemiologically well-posed in $D$ [4], and it is sufficient to consider solutions in $D$.

\section{Model Equilibrium States}

Solving the system of equations in (3) at stationary points to determine the equilibrium state of the model, it is observed that the model equations gives two independent solutions which are called the state of infection free $\left(\mathrm{E}_{0}\right)$ equilibrium or state of no infection and endemic $\left(\mathrm{E}_{1}\right)$ equilibrium state given respectively as:

$$
E_{0}=\left(S_{0}, I_{0}, Q_{0}, R_{0}, M_{0}\right)=\left(\frac{\pi c_{5}}{\mu\left(c_{1}+\alpha\right)}, 0,0,0, \frac{\pi \chi}{\mu\left(c_{1}+\alpha\right)}\right)
$$

$E_{1}=\left(S_{1}, I_{1}, Q_{1}, R_{1}, M_{1}\right)$ where

$$
S_{1}=\frac{\eta \varepsilon c_{3}+\eta \gamma \omega_{2}-c_{2} c_{3} c_{4}}{c_{4}\left(\gamma \beta_{2}+c_{3} \beta_{1}\right)}
$$

$I_{1}=-\frac{\left(\Pi \gamma \beta_{2} c_{4} c_{5}+\Pi \beta_{1} c_{3} c_{4} c_{5}-\alpha \eta \gamma \chi \omega_{2}-\alpha \eta \epsilon \chi c_{3}+\alpha \chi c_{2} c_{3} c_{4}+\eta \gamma c_{1} c_{5} \omega_{2}+\eta \epsilon c_{1} c_{3} c_{5}-c_{1} c_{2} c_{3} c_{4} c_{5}\right) c_{3}}{\alpha \gamma^{2} \beta_{2} \omega_{1} \omega_{2}+\alpha \gamma \epsilon \beta_{2} c_{3} \omega_{1}+\alpha \gamma \beta_{1} c_{3} \omega_{1} \omega_{2}+\alpha \epsilon \beta_{1} c_{3}{ }^{2} \omega_{1}+\eta \gamma^{2} \beta_{2} c_{5} \omega_{2}+\eta \gamma \epsilon \beta_{2} c_{3} c_{5}+\eta \gamma \beta_{1} c_{3} c_{5} \omega_{2}+\eta \epsilon \beta_{1} c_{3}{ }^{2} c_{5}-\gamma \beta_{2} c_{2} c_{3} c_{4} c_{5}-\beta_{1} c_{2} c_{3}{ }^{2} c_{4} c_{5}}$

$Q_{1}=-\frac{\gamma\left(\Pi \gamma \beta_{2} c_{4} c_{5}+\Pi \beta_{1} c_{3} c_{4} c_{5}-\alpha \eta \gamma \chi \omega_{2}-\alpha \eta \epsilon \chi c_{3}+\alpha \chi c_{2} c_{3} c_{4}+\eta \gamma c_{1} c_{5} \omega_{2}+\eta \epsilon c_{1} c_{3} c_{5}-c_{1} c_{2} c_{3} c_{4} c_{5}\right)}{\alpha \gamma^{2} \beta_{2} \omega_{1} \omega_{2}+\alpha \gamma \epsilon \beta_{2} c_{3} \omega_{1}+\alpha \gamma \beta_{1} c_{3} \omega_{1} \omega_{2}+\alpha \epsilon \beta_{1} c_{3}{ }^{2} \omega_{1}+\eta \gamma^{2} \beta_{2} c_{5} \omega_{2}+\eta \gamma \epsilon \beta_{2} c_{3} c_{5}+\eta \gamma \beta_{1} c_{3} c_{5} \omega_{2}+\eta \epsilon \beta_{1} c_{3}{ }^{2} c_{5}-\gamma \beta_{2} c_{2} c_{3} c_{4} c_{5}-\beta_{1} c_{2} c_{3}{ }^{2} c_{4} c_{5}}$

$R_{1}=-\frac{\left(\epsilon c_{3}+\gamma \omega_{2}\right)\left(\Pi \gamma \beta_{2} c_{4} c_{5}+\Pi \beta_{1} c_{3} c_{4} c_{5}-\alpha \eta \gamma \chi \omega_{2}-\alpha \eta \epsilon \chi c_{3}+\alpha \chi c_{2} c_{3} c_{4}+\eta \gamma c_{1} c_{5} \omega_{2}+\eta \epsilon c_{1} c_{3} c_{5}-c_{1} c_{2} c_{3} c_{4} c_{5}\right)}{c_{4} \gamma_{2} \beta_{2} \omega_{1} \omega_{2}}$

$c_{4}\left(\alpha \gamma^{2} \beta_{2} \omega_{1} \omega_{2}+\alpha \gamma \epsilon \beta_{2} c_{3} \omega_{1}+\alpha \gamma \beta_{1} c_{3} \omega_{1} \omega_{2}+\alpha \epsilon \beta_{1} c_{3}{ }^{2} \omega_{1}+\eta \gamma^{2} \beta_{2} c_{5} \omega_{2}+\eta \gamma \epsilon \beta_{2} c_{3} c_{5}+\eta \gamma \beta_{1} c_{3} c_{5} \omega_{2}+\eta \epsilon \beta_{1} c_{3}{ }^{2} c_{5}-\gamma \beta_{2} c_{2} c_{3} c_{4} c_{5}-\beta\right.$
$M_{1}=-\frac{1}{\left(\gamma \beta_{2}+\beta_{1} c_{3}\right) c_{4}\left(\alpha \gamma \omega_{1} \omega_{2}+\alpha \epsilon c_{3} \omega_{1}+\eta \gamma c_{5} \omega_{2}+\eta \epsilon c_{3} c_{5}-c_{2} c_{3} c_{4} c_{5}\right)}\left(\prod \gamma^{2} \beta_{2} c_{4} \omega_{1} \omega_{2}+\Pi \gamma \epsilon \beta_{2} c_{3} c_{4} \omega_{1}+\Pi \gamma \beta_{1} c_{3} c_{4} \omega_{1} \omega_{2}+\right.$

$\Pi \epsilon \beta_{1} c_{3}^{2} c_{4} \omega_{1}+\eta^{2} \gamma^{2} \chi \omega_{2}^{2}+2 \eta^{2} \gamma \epsilon \chi c_{3} \omega_{2}+\eta^{2} \epsilon^{2} \chi c_{3}^{2}+\eta \gamma^{2} c_{1} \omega_{1} \omega_{2}^{2}+2 \eta \gamma \epsilon c_{1} c_{3} \omega_{1}^{2} \omega_{2}-2 \eta \gamma \chi c_{2} c_{3} c_{4} \omega_{2}+\eta \epsilon^{2} c_{1} c_{3}^{2} \omega_{1}$

$\left.-2 \eta \epsilon \chi c_{2} c_{3}^{2} c_{4}-\gamma c_{1} c_{2} c_{3} c_{4} \omega_{1} \omega_{2}-\epsilon c_{1} c_{2} c_{3}^{2} c_{4} \omega_{1}+\chi c_{2}^{2} c_{3}^{2} c_{4}^{2}\right)$ 


\section{Reproduction Number of the Model $\left(\mathbf{R}_{e f f}\right)$}

The two solutions of the model can better be understood with the help of effective reproduction number of the model $\left(\mathrm{R}_{e f f}\right)$ which is defined as the number of new cases of the infection that arises from introduction of a single infective into the population of largely susceptible individuals. Some of the ways of calculating this $\mathrm{R}_{\text {eff }}$ is by the use of di-graph method, next generation matrix etc. The basic reproduction number of the model was calculated at the Infection Free equilibrium $\mathrm{E}_{0}$ and obtained as:

$$
R_{e f f}=\frac{\left(\beta_{1} c_{3}+\beta_{2} \gamma\right) S_{0}}{c_{2} c_{3}}
$$

\section{Stability Analysis of the Model}

Model (1) can exhibit behavioral change called bifurcation, at a point, the equilibrium may tend towards the infection free that is, no spread of disease, whereas at some other point, it may go to endemic state (break-out of the disease in the population). To interpret the mathematical model, there are different forms of analysis, which include but not limited to bifurcation analysis and stability analysis. This study consider the stability and bifurcation analysis of the model with reference to the effective reproduction number. It is important to note that the equilibrium point of a system is stable locally if all the associated eigenvalues of its variational matrix have negative real parts otherwise it is unstable at such a point. The following propositions and theorem shall be established to obtain the stability analysis of the model.

Proposition 1: $\mathrm{R}_{e f f}<1$ implies non-existence of endemic equilibrium $E_{1}$ and $E_{0}=E_{1}$ whenever $\mathrm{R}_{\text {eff }}=1$.

Theorem 2: The infection-free equilibrium (I.F.E) of system (3) is locally asymptotically stable whenever $\mathrm{R}_{\text {eff }}<1$ and unstable otherwise.

Proof: The Jacobian matrix of system (3) shall be used at the I.F.E. state to establish the above theorem. This is obtained by partially differentiating the five equations with the five state variables of the model and is given as:

$$
J_{E_{0}}=\left[\begin{array}{ccccc}
-\lambda-c_{1} & -\beta_{1} S & -\beta_{2} S & 0 & \alpha \\
\lambda & \beta_{1} S-c_{2} & \beta_{2} S & \eta & 0 \\
0 & \gamma & -c_{3} & 0 & 0 \\
0 & \varepsilon & \omega_{2} & -c_{4} & 0 \\
\chi & 0 & 0 & \omega_{1} & -c_{5}
\end{array}\right]
$$

Evaluating $J_{E_{0}}$ at the I.F.E and noting that $\lambda=\beta_{1} I+\beta_{2} Q$ to obtain;

$$
J_{E_{0}}=\left[\begin{array}{ccccc}
-c_{1} & -\beta_{1} S_{0} & -\beta_{2} S_{0} & 0 & \alpha \\
0 & \beta_{1} S_{0}-c_{2} & \beta_{2} S_{0} & \eta & 0 \\
0 & \gamma & -c_{3} & 0 & 0 \\
0 & \varepsilon & \omega_{2} & -c_{4} & 0 \\
\chi & 0 & 0 & \omega_{1} & -c_{5}
\end{array}\right]
$$

Definition 1: A square matrix $\mathbf{J}$ is called a stable matrix (Hurwitz matrix) if all eigenvalues of $\mathbf{J}$ have negative real part.

Thus, following definition 1, the I.F.E is locally asymptotically stable if the above matrix have negative eigenvalues. It is sufficient to use the Trace-Determinant approach to establish the I.F.E of the model. Thus, if the trace of the Jacobian matrix $J_{E_{0}}$ is less than zero and its determinant is greater than zero, then all the eigenvalues are real and negative. Thus, obtaining the trace of the matrix above as:

$$
\operatorname{Trace}\left(J_{E_{0}}\right)=-\left(\beta_{1} S_{0}+c_{1}+c_{2}+c_{3}+c_{4}+c_{5}\right)
$$

Which is obviously negative since all the parameters of the model are non-negative. 
The determinant of the matrix is given as:

$$
\begin{aligned}
& \left|J_{E_{0}}\right|=-\alpha \chi \gamma S_{0} \beta_{2} c_{4}-\alpha \chi S_{0} \beta_{1} c_{3} c_{4}+\gamma S_{0} \beta_{2} c_{1} c_{4} c_{5}+S_{0} \beta_{1} c_{1} c_{3} c_{4} c_{5}-\alpha \chi \epsilon \eta c_{3}-\alpha \chi \eta \gamma \omega_{2}+\alpha \chi c_{2} c_{3} c_{4}+ \\
& \quad \epsilon \eta c_{1} c_{3} c_{5}+\eta \gamma c_{1} c_{5} \omega_{2}-c_{1} c_{2} c_{3} c_{4} c_{5}
\end{aligned}
$$

Re-arranging and simplifying to get

$$
\begin{aligned}
& \left|J_{E_{0}}\right|=-\alpha \chi \gamma S_{0} \beta_{2} c_{4}-\alpha \chi S_{0} \beta_{1} c_{3} c_{4}+\alpha \chi c_{2} c_{3} c_{4}+\gamma S_{0} \beta_{2} c_{1} c_{4} c_{5}+S_{0} \beta_{1} c_{1} c_{3} c_{4} c_{5}-c_{1} c_{2} c_{3} c_{4} c_{5}+\epsilon \eta c_{1} c_{3} c_{5}+ \\
& \alpha \chi \epsilon \delta_{2} \omega_{1}+\alpha \chi \epsilon \omega_{1} \omega_{2}+\alpha \chi \eta \gamma \mu+\alpha \chi \eta \gamma \delta_{2}+\alpha \chi \eta \gamma \omega_{2}+\alpha \chi \eta \mu^{2}+\alpha \chi \eta \mu \delta_{1}+\alpha \chi \eta \mu \delta_{2}+\alpha \chi \eta \mu \omega_{2}+ \\
& \alpha \chi \eta \delta_{1} \delta_{2}+\alpha \chi \eta \delta_{1} \omega_{2}+\alpha \chi \gamma \mu^{2}+\alpha \chi \gamma \mu \delta_{2}+\alpha \chi \gamma \mu \omega_{1}+\alpha \chi \gamma \mu \omega_{2}+\alpha \chi \gamma \delta_{2} \omega_{1}+\alpha \chi \gamma \omega_{1} \omega_{2}+\alpha \chi \mu^{3}+ \\
& \alpha \chi \mu^{2} \delta_{1}+\alpha \chi \mu^{2} \delta_{2}+\alpha \chi \mu^{2} \omega_{1}+\alpha \chi \mu^{2} \omega_{2}+\alpha \chi \mu \delta_{1} \delta_{2}+\alpha \chi \mu \delta_{1} \omega_{1}+\alpha \chi \mu \delta_{1} \omega_{2}+\alpha \chi \mu \delta_{2} \omega_{1}+\alpha \chi \mu \omega_{1} \omega_{2}+ \\
& \alpha \chi \delta_{1} \delta_{2} \omega_{1}+\alpha \chi \delta_{1} \omega_{1} \omega_{2}+\alpha \eta \gamma \mu \omega_{2}+\chi \eta \gamma \mu \omega_{2}+\alpha \chi \epsilon \mu \delta_{2}+\alpha \chi \epsilon \mu \omega_{1}+\alpha \chi \epsilon \mu \omega_{2}+\alpha \chi \epsilon \mu^{2}+\eta \gamma \mu^{2} \omega_{2}
\end{aligned}
$$

On further simplification, we obtain

$$
\begin{aligned}
& \left|J_{E_{0}}\right|=-\alpha \chi c_{2} c_{3} c_{4}\left(S_{0}\left(\frac{\beta_{2} \gamma+\beta_{1} c_{3}}{c_{2} c_{3}}\right)-1\right)+c_{1} c_{4} c_{5}\left(1-S_{0}\left(\frac{\beta_{2} \gamma+\beta_{1} c_{3}}{c_{2} c_{3}}\right)\right)+\alpha \chi \epsilon \mu \omega_{1}+\alpha \chi \epsilon \mu \delta_{2}+ \\
& \alpha \chi \epsilon \delta_{2} \omega_{1}+\alpha \chi \epsilon \omega_{1} \omega_{2}+\alpha \chi \eta \gamma \mu+\alpha \chi \eta \gamma \delta_{2}+\alpha \chi \eta \gamma \omega_{2}+\alpha \chi \eta \mu^{2}+\alpha \chi \eta \mu \delta_{1}+\alpha \chi \eta \mu \delta_{2}+\alpha \chi \eta \mu \omega_{2}+ \\
& \alpha \chi \eta \delta_{1} \delta_{2}+\alpha \chi \eta \delta_{1} \omega_{2}+\alpha \chi \gamma \mu^{2}+\alpha \chi \gamma \mu \delta_{2}+\alpha \chi \gamma \mu \omega_{1}+\alpha \chi \gamma \mu \omega_{2}+\alpha \chi \gamma \delta_{2} \omega_{1}+\alpha \chi \gamma \omega_{1} \omega_{2}+\alpha \chi \mu^{3}+ \\
& \alpha \chi \mu^{2} \delta_{1}+\alpha \chi \mu^{2} \delta_{2}+\alpha \chi \mu^{2} \omega_{1}+\alpha \chi \mu^{2} \omega_{2}+\alpha \chi \mu \delta_{1} \delta_{2}+\alpha \chi \mu \delta_{1} \omega_{1}+\alpha \chi \mu \delta_{1} \omega_{2}+\alpha \chi \mu \delta_{2} \omega_{1}+\alpha \chi \mu \omega_{1} \omega_{2}+ \\
& \alpha \chi \delta_{1} \delta_{2} \omega_{1}+\alpha \chi \delta_{1} \omega_{1} \omega_{2}+\alpha \eta \gamma \mu \omega_{2}+\chi \eta \gamma \mu \omega_{2}+\alpha \chi \epsilon \mu \omega_{2}+\alpha \chi \epsilon \mu^{2}+\eta \gamma \mu^{2} \omega_{2}
\end{aligned}
$$

$$
\begin{aligned}
& \left|J_{E_{0}}\right|=-\alpha \chi c_{2} c_{3} c_{4}\left(R_{e f f}-1\right)+c_{1} c_{4} c_{5}\left(1-R_{e f f}\right)+\alpha \chi \epsilon \mu \omega_{1}+\alpha \chi \epsilon \mu \delta_{2}+\alpha \chi \epsilon \delta_{2} \omega_{1}+\alpha \chi \epsilon \omega_{1} \omega_{2}+ \\
& \alpha \chi \eta \gamma \mu+\alpha \chi \eta \gamma \delta_{2}+\alpha \chi \eta \gamma \omega_{2}+\alpha \chi \eta \mu^{2}+\alpha \chi \eta \mu \delta_{1}+\alpha \chi \eta \mu \delta_{2}+\alpha \chi \eta \mu \omega_{2}+\alpha \chi \eta \delta_{1} \delta_{2}+\alpha \chi \eta \delta_{1} \omega_{2}+ \\
\Rightarrow & \alpha \chi \gamma \mu^{2}+\alpha \chi \gamma \mu \delta_{2}+\alpha \chi \gamma \mu \omega_{1}+\alpha \chi \gamma \mu \omega_{2}+\alpha \chi \gamma \delta_{2} \omega_{1}+\alpha \chi \gamma \omega_{1} \omega_{2}+\alpha \chi \mu^{3}+\alpha \chi \mu^{2} \delta_{1}+\alpha \chi \mu^{2} \delta_{2}+ \\
& \alpha \chi \mu^{2} \omega_{2}+\alpha \chi \mu \delta_{1} \delta_{2}+\alpha \chi \mu \delta_{1} \omega_{1}+\alpha \chi \mu \delta_{1} \omega_{2}+\alpha \chi \mu \delta_{2} \omega_{1}+\alpha \chi \mu \omega_{1} \omega_{2}+\alpha \chi \delta_{1} \delta_{2} \omega_{1}+\alpha \chi \delta_{1} \omega_{1} \omega_{2}+ \\
& \alpha \eta \gamma \mu \omega_{2}+\chi \eta \gamma \mu \omega_{2}+\alpha \chi \epsilon \mu \omega_{2}+\alpha \chi \epsilon \mu^{2}+\eta \gamma \mu^{2} \omega_{2}+\alpha \chi \mu^{2} \omega_{1}
\end{aligned}
$$

Which is strictly positive whenever $\mathrm{R}_{e f f}<1$. Thus, the I.F.E is locally asymptotically stable whenever $\mathrm{R}_{e f f}<1$ and unstable otherwise.

\section{Global Stability Analysis of the Model at Infection Free Equi- librium}

We adopt Castillo-Chavez et al., [12] to obtain the global stability analysis of the new generalized SIQRM tuberculosis model. Suppose:

$$
\begin{aligned}
& \frac{d X}{d t}=F(X, Z) \\
& \frac{d Z}{d t}=G(X, Z), \quad G(X, 0)=0
\end{aligned}
$$

where both $X \in \Re^{m}$ and $Z \in \Re^{n}$ and their components denote the number of uninfected and infected individuals respectively and $E_{0}=\left(x^{*}, 0\right)$ is the infection free equilibrium point. Then, the fixed point $E_{0}=\left(x^{*}, 0\right)$ is a globally asymptotic stable (g.a.s.) equilibrium of the system (3) provided that $R_{e f f}<1$ (l.a.s.) and that assumptions $(H l)$ and $(H 2)$ below are satisfied: 
(H1) For $\frac{d X}{d t}=F(X, 0), \quad X^{*}$ is globally asymptotically stable (g.a.s.)

(H2) $G(X, Z)=A Z-\hat{G}(X, Z), \quad \hat{G}(X, Z) \geq 0$ for $(X, Z) \in \Omega$

where $A=D_{z} G\left(X^{*}, 0\right)$ is an $M$-matrix (the off diagonal elements of $A$ are non-negative) and 0 is the region where the model makes biological sense.

Theorem 3: The equilibrium point $E_{0}=\left(W^{*}, 0\right)$ of system (3) is Globally Asymptotically Stable provided that $R_{e f f}<1$ and unstable if otherwise.

Proof: It is sufficient to establish the two criteria from Castillo-Chavez et al., (2002) to establish the globally asymptotically stable (g.a.s.) equilibrium for the system (3). Splitting system of equations (3) into:

$$
\begin{gathered}
X=(S, R, M), \quad Z=(I, Q) \\
\frac{d X}{d t}=F(X, Z)=\left(\begin{array}{c}
\pi+\alpha M-\lambda S-c_{1} S \\
\epsilon I+\omega_{2} Q-c_{4} R \\
\omega_{1} R+\chi S-c_{5} M
\end{array}\right) \\
F(X, 0)=\left(\begin{array}{c}
\pi+\alpha M-c_{1} S \\
0 \\
\chi S-c_{5} M
\end{array}\right)
\end{gathered}
$$

Solving the resulting equation in (15) at equilibrium to obtain

$$
F(X, 0)=E_{0}=\left(S_{0}, R_{0}, M_{0}\right)=\left(\frac{\pi c_{5}}{\mu\left(c_{1}+\alpha\right)}, 0, \frac{\pi \chi}{\mu\left(c_{1}+\alpha\right)}\right)
$$

Also, from (15),

$$
\begin{aligned}
& \frac{d S}{d t}=\pi+\alpha M-c_{1} S \\
& \Rightarrow \frac{d S}{d t} \leq \pi-c_{1} S \\
& \therefore S(t) \leq \frac{\pi}{\mu}+\left(S(0)-\frac{\pi}{\mu}\right) e^{-\mu t}
\end{aligned}
$$

Obviously, as;

$$
t \rightarrow \infty, \quad S(t) \rightarrow \frac{\pi}{\mu}
$$

Similarly, solving for $M$ from (15) to obtain $M(t) \leq k e^{-c_{5} t}$ which approaches a constant value as $t \rightarrow \infty$. Thus, condition $H 1$ is satisfied. Now,

$$
G(X, Z)=\left[\begin{array}{c}
\lambda S+\eta R-c_{2} I \\
\gamma I-c_{3} Q
\end{array}\right]=\left[\begin{array}{c}
\left(\beta_{1} I+\beta_{2} Q\right) S+\eta R-c_{2} I \\
\gamma I-c_{3} Q
\end{array}\right]
$$

Put $A=D_{z} G\left(X^{*}, 0\right)$ (the Jacobian matrix of the infected compartment at the infection free equilibrium state),

$$
\begin{aligned}
A & =\left[\begin{array}{cc}
\beta_{1} S_{0}-c_{2} & \beta_{2} S_{0} \\
\gamma & -c_{3}
\end{array}\right] \\
A Z & =\left[\begin{array}{cc}
\beta_{1} S_{0}-c_{2} & \beta_{2} S_{0} \\
\gamma & -c_{3}
\end{array}\right]\left[\begin{array}{l}
I \\
Q
\end{array}\right] \\
& =\left[\begin{array}{c}
\left(\beta_{1} S_{0}-c_{2}\right) I+\beta_{2} S_{0} Q \\
\gamma I-c_{3} Q
\end{array}\right]
\end{aligned}
$$


From $H 1, \quad S_{0} \rightarrow \frac{\pi}{\mu}=N$

$$
\begin{aligned}
& A Z=\left[\begin{array}{c}
\beta_{1} N I-c_{2} I+\beta_{2} N Q \\
\gamma I-c_{3} Q
\end{array}\right] \\
& \hat{G}(X, Z)=A Z-G(X, Z) \\
& \hat{G}(X, Z)=\left[\begin{array}{c}
\beta_{1} N I-c_{2} I+\beta_{2} N Q \\
\gamma I-c_{3} Q
\end{array}\right]-\left[\begin{array}{c}
\left(\beta_{1} I+\beta_{2} Q\right) S+\eta R-c_{2} I \\
\gamma I-c_{3} Q
\end{array}\right]
\end{aligned}
$$

At infection free equilibrium, $R_{0}=0$. Using this for further simplification, we have;

$$
\begin{aligned}
& \hat{G}(X, Z)=\left[\begin{array}{c}
\left(\beta_{1} I+\beta_{2} Q\right)(N-S) \\
0
\end{array}\right] \\
& =\left[\begin{array}{c}
\left(\beta_{1} I+\beta_{2} Q\right) N\left(1-\frac{S}{N}\right) \\
0
\end{array}\right] \geq 0
\end{aligned}
$$

Since $0 \leq S \leq N$, it is clear that $\hat{G}(X, Z) \geq 0$. And since $E_{0}$ is G.A.S. equilibrium of $\frac{d X}{d t}$ from $H 1$, it follows that $E_{0}$ is Globally Asymptotically Stable (G.A.S)

\section{Bifurcation Analysis of the Model}

The stability analysis of an equilibrium point determines whether a system remain near the equilibrium point when subjected to slight adjustment (or perturbation). Thus, the solution of an epidemic models are considered reasonable when small perturbation does not lead to large variation. In this section, we shall be considering the bifurcation analysis of the SIQRM tuberculosis model at infection free equibrium by using centre manifold theory as applied by Mukandavire et al., 2009 [7]. For easy representation, let $S=x_{1}, \quad I=x_{2}, \quad Q=x_{3}, \quad R=x_{4}$ and $M=x_{5}$, then system (3) becomes:

$$
\begin{aligned}
& f_{1}=\frac{d x_{1}}{d t}=\pi+\alpha x_{5}-\lambda x_{1}-c_{1} x_{1} \\
& f_{2}=\frac{d x_{2}}{d t}=\lambda x_{1}+\eta x_{4}-c_{2} x_{2} \\
& f_{3}=\frac{d x_{3}}{d t}=\gamma x_{2}-c_{3} x_{3} \\
& f_{4}=\frac{d x_{4}}{d t}=\epsilon x_{2}+\omega_{2} x_{3}-c_{4} x_{4} \\
& f_{5}=\frac{d x_{5}}{d t}=\omega_{1} x_{4}+\chi x_{1}-c_{5} x_{5}
\end{aligned}
$$

Using (6) and (17), the bifurcation parameter is chosen at $R_{\text {eff }}=1$ as:

$$
\beta=\beta^{*}=\frac{c_{2} c_{3} \mu}{\pi\left(\beta_{1} c_{3}+\beta_{2} \gamma\right)}
$$

The matrix (8) has a right eigenvector denoted by $U=\left(u_{1}, u_{2}, u_{3}, u_{4}, u_{5}\right)^{T}$ as follows:

$$
J_{\text {Right }}=\left(\begin{array}{ccccc}
-c_{1} & \frac{-\beta_{1}^{*} \pi}{\mu} & \frac{-\beta_{2}^{*} \pi}{\mu} & 0 & \alpha \\
0 & \frac{\beta_{1}^{*} \pi}{\mu}-c_{2} & \frac{\beta_{2}^{*} \pi}{\mu} & \eta & 0 \\
0 & \gamma & -c_{3} & 0 & 0 \\
0 & \varepsilon & \omega_{2} & -c_{4} & 0 \\
\chi & 0 & 0 & \omega_{1} & -c_{5}
\end{array}\right)\left(\begin{array}{c}
u_{1} \\
u_{2} \\
u_{3} \\
u_{4} \\
u_{5}
\end{array}\right)=0
$$


Solving (23) to get,

$$
\begin{aligned}
& u_{2}>0 \quad(\text { free }), u_{3}=\frac{\gamma u_{2}}{c_{3}}, u_{4}=\left(\frac{\epsilon c_{3}+\omega_{2} \gamma}{c_{3} c_{4}}\right) u_{2} \\
& u_{1}=\frac{c_{4} c_{5}\left(c_{3} \beta_{1}^{*} \pi+\beta_{2}^{*} \pi \gamma\right)-\mu \omega_{1} \alpha\left(\epsilon c_{3}+\omega_{2} \gamma\right)}{\mu c_{3} c_{4}} \\
& u_{5}=\frac{\chi c_{4} c_{5}\left(c_{3} \beta_{1}^{*} \pi+\beta_{2}^{*} \pi \gamma\right)+(1-\alpha) \mu \omega_{1}\left(\epsilon c_{3}+\omega_{2} \gamma\right)}{\mu c_{3} c_{4}}
\end{aligned}
$$

Similarly, the left eigenvector $V=\left(v_{1}, v_{2}, v_{3}, v_{4}, v_{5}\right)$ is obtained from the solution of:

$$
J_{\text {Left }}=\left(\begin{array}{lllll}
v_{1} & v_{2} & v_{3} & v_{4} & v_{5}
\end{array}\right)\left(\begin{array}{ccccc}
-c_{1} & \frac{-\beta_{1}^{*} \pi}{\mu} & \frac{-\beta_{2}^{*} \pi}{\mu} & 0 & \alpha \\
0 & \frac{\beta_{1}^{*} \pi}{\mu}-c_{2} & \frac{\beta_{2}^{*} \pi}{\mu} & \eta & 0 \\
0 & \gamma & -c_{3} & 0 & 0 \\
0 & \varepsilon & \omega_{2} & -c_{4} & 0 \\
\chi & 0 & 0 & \omega_{1} & -c_{5}
\end{array}\right)=0
$$

as:

$$
\begin{aligned}
& v_{1}>0 \quad \text { free, } v_{5}=\frac{c_{1} v_{1}}{\chi}, \quad v_{2}=\left(\frac{c_{5} y_{6}+\gamma y_{8}}{c_{5} y_{5}+\gamma y_{7}}\right) v_{1} \\
& v_{4}=\frac{1}{c_{4}}\left(\frac{\eta\left(c_{5} y_{6}+\gamma y_{8}\right)}{c_{5} y_{5}+\gamma y_{7}}+\frac{\omega_{1} c_{1} v_{1}}{\chi}\right) v_{1} \\
& v_{3}=\frac{1}{\gamma}\left[y_{6}+y_{5}\left(\frac{c_{5} y_{6}+\gamma y_{8}}{c_{5} y_{5}+\gamma y_{7}}\right)\right] v_{1}
\end{aligned}
$$

where $y_{5}=\frac{\beta_{1}^{*} \pi}{\mu}-c_{2}+\frac{\eta \epsilon}{c_{4}}, y_{6}=\frac{\beta_{1}^{*} \pi}{\mu}+\frac{\epsilon \omega_{1} c_{1}}{c_{4} \chi}, y_{7}=\frac{\beta_{2}^{*} \pi}{\mu}+\frac{\omega_{2} \eta}{c_{4}}$ and $y_{8}=\frac{\beta_{2}^{*} \pi}{\mu}+\frac{\omega_{1} c_{1}}{c_{4} \chi}$

According to Centre Manifold Theory (CMT), as applied by Mukandavire et al., (2009), the dynamics of equilibrium of system (3) depend solely on $a$ and $b$ given as:

$$
\begin{aligned}
& a=\sum_{k, i, j=1}^{n} v_{k} u_{i} u_{j} \frac{\partial g_{k}(0,0)}{\partial x_{i} \partial x_{j}} \\
& b=\sum_{k, i, j=1}^{n} v_{k} u_{i} \frac{\partial g_{k}(0,0)}{\partial x_{i} \partial \phi}
\end{aligned}
$$

The non-zero second order partial derivative of (3) at I.F.E is given in equation (28) below:

$$
\begin{array}{rlrl}
\frac{\partial^{2} f_{1}}{\partial x_{1} \partial x_{2}} & =\frac{\partial^{2} f_{1}}{\partial x_{2} \partial x_{1}}=-\beta_{1} ; & \frac{\partial^{2} f_{1}}{\partial x_{2} \partial \beta_{1}}=\frac{\partial^{2} f_{1}}{\partial \beta_{1} \partial x_{2}}=-\frac{\pi}{\mu} \\
\frac{\partial^{2} f_{1}}{\partial x_{1} \partial x_{3}}=\frac{\partial^{2} f_{1}}{\partial x_{3} \partial x_{1}}=-\beta_{2} ; & \frac{\partial^{2} f_{1}}{\partial x_{3} \partial \beta_{2}}=\frac{\partial^{2} f_{1}}{\partial \beta_{2} \partial x_{3}}=-\frac{\pi}{\mu} \\
\frac{\partial^{2} f_{2}}{\partial x_{1} \partial x_{2}}=\frac{\partial^{2} f_{2}}{\partial x_{2} \partial x_{1}}=\beta_{1} ; & \frac{\partial^{2} f_{2}}{\partial x_{2} \partial \beta_{1}}=\frac{\partial^{2} f_{2}}{\partial \beta_{1} \partial x_{2}}=\frac{\pi}{\mu} \\
\frac{\partial^{2} f_{2}}{\partial x_{1} \partial x_{3}}=\frac{\partial^{2} f_{2}}{\partial x_{3} \partial x_{1}}=\beta_{2} ; & \frac{\partial^{2} f_{2}}{\partial x_{3} \partial \beta_{2}}=\frac{\partial^{2} f_{2}}{\partial \beta_{2} \partial x_{3}}=\frac{\pi}{\mu}
\end{array}
$$

Substituting (24), (26) and (28) into (27) with further simplification to obtain:

$$
\begin{aligned}
& a=\frac{-2 v_{1} u_{2}^{2} \gamma \beta^{*}\left[\pi c_{4}\left(c_{5} \beta_{1}+\gamma \beta_{2} \chi\right)+\omega_{1} c_{1}\left(c_{5}+\gamma \mu\right)\right]}{\chi \mu c_{3} c_{4}}<0 \\
& b=\frac{v_{1} u_{2} \pi}{\mu}>0
\end{aligned}
$$


Whenever $a<0$ and $b>0$, by CMT, $\phi$ changes from negative to positive, the equilibrium point 0 changes its stability from stable to unstable. Correspondingly, a negative unstable equilibrium becomes positive and locally asymptotically stable. Hence, the model exhibits forward bifurcation when $R_{\text {eff }}=1$. The implication of bifurcation is that the classical requirement of having the basic reproduction number less than unity, although necessary, is no longer sufficient to curtail the spread of the disease within the host community.

\section{Numerical simulation and discussion}

The SIQRM model developed for analysis in this work is an entirely new model for tuberculosis disease. Thus, the numerical analysis of the result obtained was presented in this section using secondary data obtained from literature. Different initial values for all the state variables are used to obtain the graphs while the values of the parameters used are as stated in Table 1 . The initial populations were chosen so that the total population $N(t)=S(t)+I(t)+Q(t)+R(t)+M(t)=6000$ The population for each curve are chosen as follows:

Curve 1: $\mathrm{S}(\mathrm{t})=5000, \mathrm{I}(\mathrm{t})=200, \mathrm{Q}(\mathrm{t})=100, \mathrm{R}(\mathrm{t})=200$ and $\mathrm{M}(\mathrm{t})=500$ while curve 2 has 3500 , $1200,400,300$ and 600 respectively, curve 3: 3000, 1000, 500, 600 and 900 respectively and curve 4 has 4500,300, 200, 300 and 700 respectively. The graph for each compartment is plotted and the result were given below:

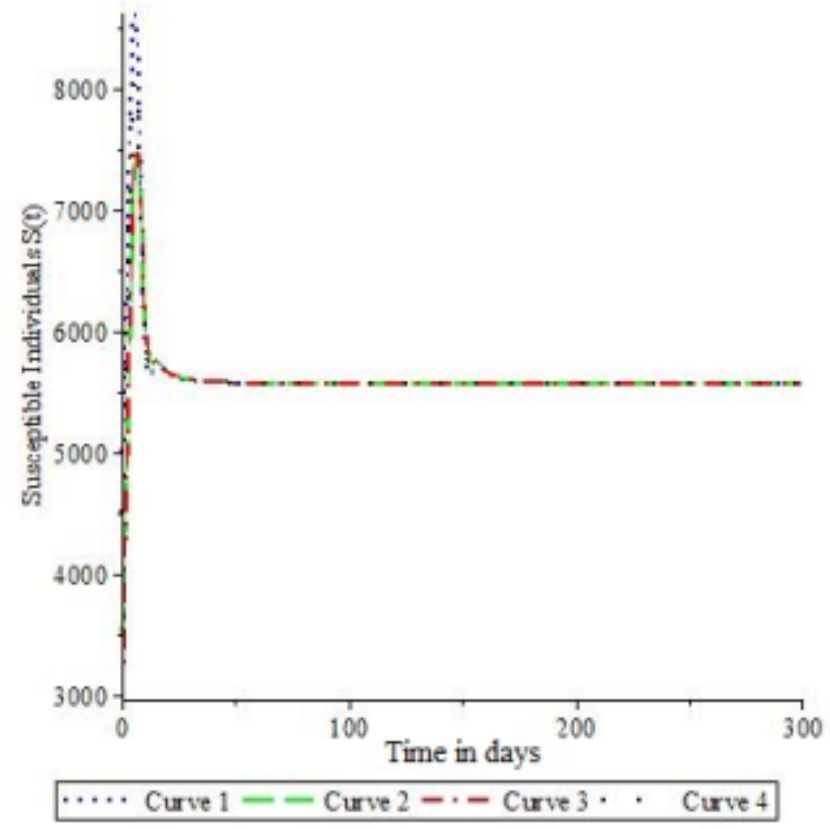

Figure 2: Change in S-compartment

The above figure 2 showed the rate of change in compartment S-population as time increases. The population first increase, then it decreases until it reached the equilibrium point. 


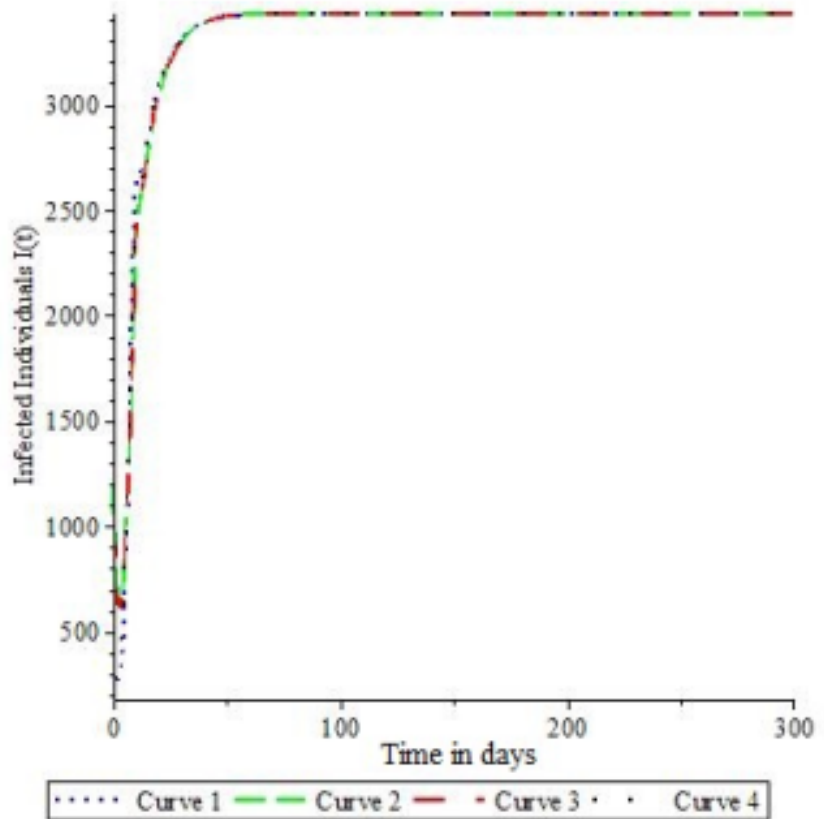

Figure 3: Change in I-compartment

The introduction of an infected individual alters the dynamics of any population as long as the reproduction number of the disease is greater than unity. This is obvious from the figure 3 as I-compartment increases in population until an equilibrium position is obtained.

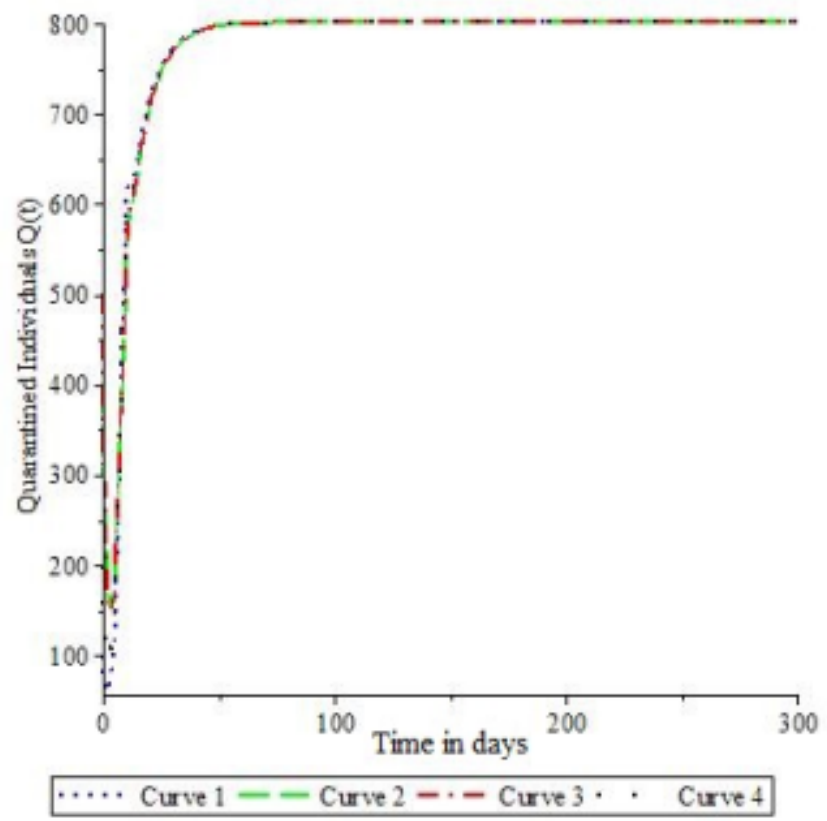

Figure 4: Change in Q-compartment

The more cases of infection reported, the more the removal of people exposed, which is the reason while figure 4 has an upward trend until the equilibrium is reached. 


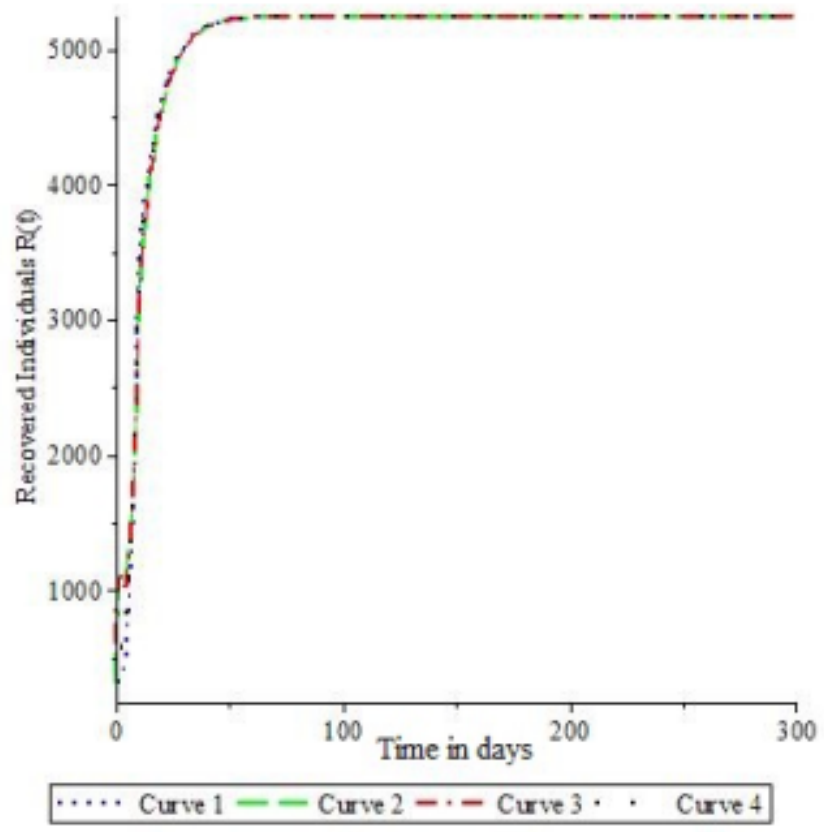

Figure 5: Change in R-compartment

Recovered individuals increases due to presence of effective treatment until the equilibrium point is attained as can be seen in the figure 5 above.

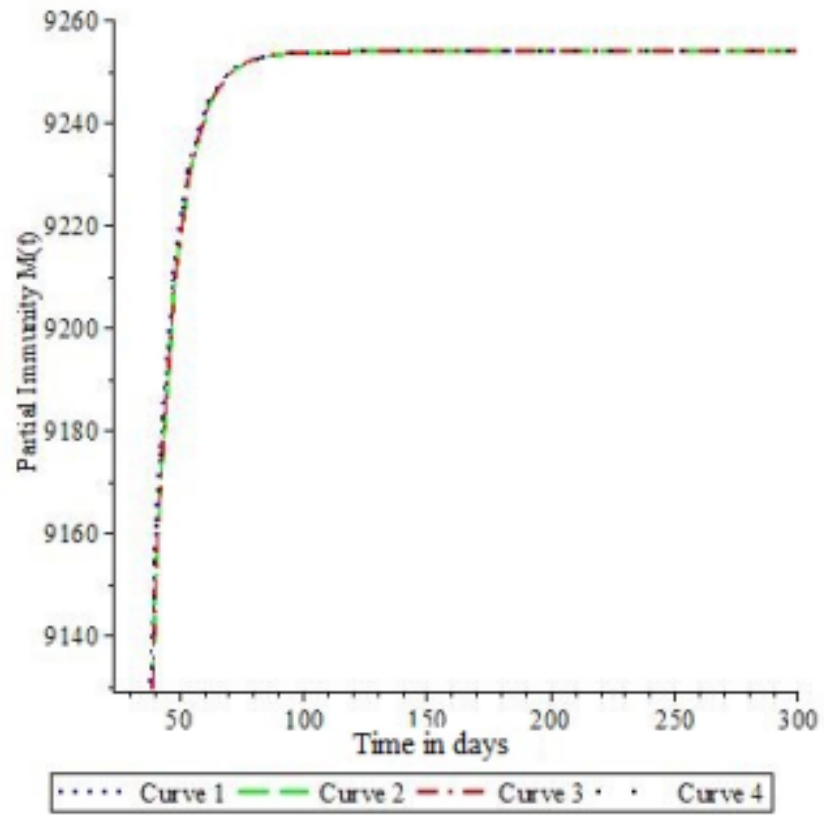

Figure 6: Change in M-compartment

The number of immune individuals increases with time due to immunity obtained from vaccine as well as partial immunity obtained from effective treatment of the disease. 


\section{Conclusion}

A five-compartmental deterministic mathematical model for the transmission of infectious disease in the presence of vaccination, immunity loss and relapse is formulated and analyzed at the disease free equilibrium state. The model reproduction number was obtained and used to establish the local stability analysis of the model at DFE. Simulation was done and the findings of the study include:

1. Two equilibria states exist for the model which are the disease free and endemic equilibria points

2. Whenever the reproduction number of the disease is less than unity, the disease cannot spread within the community.

3. In the presence of effective treatment, the number of individual with immunity increases due to boost in the anti-bodies.

4. Quarantine can only be effective if people are well sensitized about the sign and symptoms of the infection, else the infection will continue to rise within the host community.

\section{Acknowledgements}

The authors are grateful to the authors referred in this work.

\section{References}

[1] Akinyemi, S. T., Ibrahim, M. O., Usman, I. G. and Odetunde O. (2016). Global Stability Analysis of Sir Epidemic Model with Relapse and Immunity Loss. International Journal of Applied Science and Mathematical Theory, 2(1): 1-14.

[2] Berec, L. (2010). Modeling Infectious Diseases in Humans and Animals. Czech Republic: Department of Mathematics and Biomathematics, Faculty of Science, University of South Bohemia, Brani'sovsk 31, 37005, 1-58.

[3] Daabo, M. I., Makinde O. D. and Baba S. (2013). Modeling the combined effects of careless susceptible and infective immigrants on the transmission dynamics of HIV/AIDS epidemics. Journal of Public Health and Epidemiology 5(9):362-369

[4] Hethcote, H. W. (2000). The mathematics of infectious diseases. SIAM Review, 42, 599-653.

[5] Egonmwan, A. O. and Okuonghae, D. (2018). Analysis of a mathematical model for tuberculosis with diagnosis. Journal of Applied Mathematics and Computing DOI: 10.1007/s12190-018$1172-1$

[6] Fors J, Strydom N, Fox WS, Keizer RJ and Savic RM (2020). Mathematical model and tool to explore shorter multi-drug therapy options for active pulmonary tuberculosis. PLoS Computational Biology 16(8): e1008107. https://doi.org/10.1371/journal.pcbi.1008107

[7] Mukandavire, Z.,Gumel, A.B., Garira, W. and Tchuenche, J. M. (2009). Mathematical analysis of a model of HIV-Malaria coinfection. Mathematical Biosciences and Engineering, 6(2), 333 362. doi:10.3934/mbe.2009.6.333

[8] Okuonghae, D. (2013). A mathematical model of tuberculosis transmission with heterogeneity in disease susceptibility and progression under a treatment regime for infectious cases. Applied Mathematical Modelling, 37(s 10-11):6786-6808. DOI: 10.1016/j.apm.2013.01.039 
[9] Portal, M. (2014). A Dictionary of Epidemiology. New York: Oxford University Press.

[10] World Health Organization (2020). Global Tuberculosis Report 2020. Available online at: https://www.who.int/teams/global-tuberculosis-programme/tb-reports/global-tuberculosisreport- 2020

[11] Mettle, F.O, Affi, P.O. and Twumasi, C. (2020). Modelling the Transmission Dynamics of Tuberculosis in the Ashanti Region of Ghana. Interdiscip Perspect Infect Dis. 2020:4513854. doi: 10.1155/2020/4513854. PMID: 32318105; PMCID: PMC7150731.

[12] Castillo-Chavez, C., Feng, Z. and Huang, W. (2002). On the computation of $R_{0}$ and its role on global stability. The IMA Volumes in Mathematics and its Applications. Available online at: https://ecommons.cornell.edu/bitstream/handle/1813/32146/BU-1553-M.pdf?sequence=1. 